\title{
Article \\ Algebra of Symmetry Operators for Klein-Gordon-Fock Equation
}

\author{
Valeriy V. Obukhov ${ }^{1,2}$
}

1 Institute of Scientific Research and Development, Tomsk State Pedagogical University, 60 Kievskaya St., 634041 Tomsk, Russia; obukhov@tspu.edu.ru

2 Laboratory for Theoretical Cosmology, Tomsk State University of Control Systems and Radio Electronics, 36 Lenin Avenue, 634050 Tomsk, Russia

\begin{abstract}
All external electromagnetic fields in which the Klein-Gordon-Fock equation admits the first-order symmetry operators are found, provided that in the space-time $V_{4}$ a group of motion $G_{3}$ acts simply transitively on a non-null subspace of transitivity $V_{3}$. It is shown that in the case of a Riemannian space $V_{n}$, in which the group $G_{r}$ acts simply transitively, the algebra of symmetry operators of the $n$-dimensional Klein-Gordon-Fock equation in an external admissible electromagnetic field coincides with the algebra of operators of the group $G_{r}$.
\end{abstract}

Keywords: Klein-Gordon-Fock equation; algebra of symmetry operators; theory of symmetry; separation of variables; linear partial differential equations

check for

updates

Citation: Obukhov, V.V. Algebra of Symmetry Operators for Klein-Gordon-Fock Equation.

Symmetry 2021, 13, 727.

https://doi.org/10.3390/ sym13040727

Academic Editor: Sergei D. Odintsov

Received: 31 March 2021

Accepted: 15 April 2021

Published: 20 April 2021

Publisher's Note: MDPI stays neutral with regard to jurisdictional claims in published maps and institutional affiliations.

Copyright: (C) 2021 by the author. Licensee MDPI, Basel, Switzerland. This article is an open access article distributed under the terms and conditions of the Creative Commons Attribution (CC BY) license (https:// creativecommons.org/licenses/by/ $4.0 /)$.

\section{Introduction}

The Klein-Gordon-Fock equation describes the dynamics of a charged massive scalar particle interacting with an electromagnetic field. Recently, interest in the Klein-GordonFock equation has grown significantly due to attempts to solve the problem of dark matter in the framework of the scalar-tensor theory. For the successful construction of a realistic model, it is especially important to have, if not an exact solution of the basic equation, then at least a reliable approximate solution. This possibility was provided by the method of complete separation of variables, which makes it possible to reduce the original equations in partial variables to systems of ordinary differential equations. In this case, obtaining approximate solutions is not a problem, while traditional approaches in the framework of perturbation theory may turn out to be ineffective. We should note that when finding all known exact solutions of the gravitational field equations (including self-consistent ones) on certain stages, there is always a separation of variables. The symmetry of the classical and quantum one-particle motion equations is closely related to the symmetry of the space itself. If space admits sets of geometric objects consisting of the Killing fields, then the motion equations also have symmetry operators defined by the same sets. From a physical point of view, sets of three geometric objects are of particular interest. In the Stackel spaces, such sets are called complete. They consist of mutually commuting Killing vector and tensor fields. Homogeneous spaces are other interesting types of spaces with three geometric objects. In them, the group of motion $G_{3}$ acts simply transitively on a non-null hypersurface of transitivity. The theory of complete separation of variables (or the theory of the Stackel spaces) is a consequence of the theory of symmetry. The complete separation of variables in the classical, and under certain conditions, in the quantum motion equations for a test particle is possible only in the Stackel space. The Stackel spaces were named after Paul Stackel, who gave the first example of such space [1]. Besides Stackel, useful and applicable contributions to the construction of the theory were made by Levi-Civita [2], Yarov-Yarovoy [3], and Shapovalov [4-6]. V. V. Shapovalov proved the main theorem of the theory of Stackel spaces. The theorem makes it possible to carry out an invariant partition of the of the Stackel metrics set into equivalence classes. This made it possible to solve 
classification problems separately for each type of space (to list all nonequivalent metrics and electromagnetic potentials).

In paper [7], the theory was generalized to the case of complex privileged coordinate systems. A more detailed description of the theory and a fairly detailed bibliography can be found in the works [8-10]. Shapovalov's theorem is of great theoretical and applied relevance. In particular, the theorem made it possible to obtain and systematize all cases of complete separation of variables in one-particle equations of classical and quantum mechanics in flat space-time. A large number of papers have been devoted to the theory of complete separation of variables since its inception. Nevertheless, it can be considered complete only for the free Hamilton-Jacobi equation. After the publication of articles [10-13] the classification problem of complete separation of variables for the Hamilton-Jacobi equation in an external electromagnetic field has been solved. However, even for the free Klein-Gordon-Fock equation, the problem of constructing and classifying the Stackel spaces is still topical, since the equations that define, according to Shapovalov's theorem, the necessary and sufficient conditions for the complete separation of variables have not yet been solved in a general case. Moreover, they have not been solved for the free Dirac-Fock equation, as well as for all quantum equations of motion in the external fields of a gauge nature. Only isolated results have been obtained. For example, the problem of complete separation of variables in the Klein-Gordon-Fock equation has been solved for the Einstein spaces and for vacuum solutions of the Einstein equations (see [14-17]). In the papers [18-20] intersections of sets of homogeneous spaces and the Stackel spaces have been considered. Due to the high level of symmetry of the Stackel spaces, they remain interesting objects for research in various branches of theoretical and mathematical physics. An important direction is associated with the study of geometry and physics in spaces and fields that admit complete separation of variables in quantum equations of motion (see for, example, [21-25]).

Solutions of scalar equations are widely used in cosmology, including in the study of the of dark matter and dark energy problem (see, for example, [26]). Methods of the symmetry theory of are used to justify the choice of models of the extended gravity cosmology (see, for example, [27]), and to find realistic models [28,29], etc.

Let us note one more feature of the Stackel spaces. The presence vector and tensor Killing fields in space-time allows separating variables in the Einstein equation, since in a privileged coordinate system the metric contains only functions, arbitrarily depending on one of the non-privileged variables. The fact that the metric is given with the indicated arbitrariness makes it possible to reduce the problem of integrating field equations and equations of motion to the problem of solving functional equations and, as a consequence, to the problem of integrating systems of ordinary differential equations. The same arbitrary nature is peculiar for space-time manifold $V_{4}$, when a group of motions $G_{3}$ acts simply transitive on a subspace $V_{3}$.

The gravitational equations for the space-time manifolds with such groups also admit the separation of variables, and the solution of the field equations is reduced to the classification of the energy-momentum tensor of matter admitted by a given geometry. The methods of the complete separation of variables theory in these spaces are generally inapplicable. For them, it is possible to use the method of linear partial differential equations integration, developed in [30], using non-commutative algebras of symmetry operators. The method made it possible to significantly expand the classification of external fields and Riemannian manifolds that admit the existence of exact solutions of the Klein-Gordon-Fock equation, and served as the foundation for the study of quantum effects in homogeneous spaces. In particular, in [31-33], a complete classification of spaces admitting a simply transitive action of the motions groups $G_{4}$ was obtained, provided that the Klein-Gordon-Fock equation is exactly solved by non-commutative integration methods. In [34-38], a similar problem was solved for Dirac-Fock equation.

In this article, a complete classification of admissible electromagnetic fields is carried out for the case when the groups of motions $G_{3}$ act simply transitively on the nonzero 
subspace $V_{3}$ of the space-time manifold $V_{4}$. All the corresponding electromagnetic fields for such groups are found. By admissible we mean fields for which the Klein-Gordon-Fock equation admits symmetry operators.

Let us note the following circumstances. As it was already noted, all external electromagnetic fields in which the Hamilton-Jacobi equation admits complete separation of variables for the test charge were found [10-13]. Thus, the problem under consideration can be viewed as an extension of the work, the final goal of which is to classify all admissible external electromagnetic fields, both with respect to the action of the symmetry operators of the complete set and with respect to the action of the operators of the group $G_{r}$.

\section{Conditions for the Existence of Symmetry Operators}

Consider a Riemannian space $V_{n}$. in which a group $G_{r}$ acts simply transitively on a subspace $V_{r}$. Coordinate indexes of variables in the local coordinate system $\left[u^{i}\right]$ of the space $V_{n}$ will be denoted as follows: $i, j, k, l=0,1, \ldots n-1$. The transitivity subspace where the group $G_{r}$ acts is given by the system of equations:

$$
\psi^{p}\left(u^{i}\right)=\text { const },(p, q=0, \ldots, n-r-1) .
$$

In what follows, it is assumed that $\psi^{p}=u^{p}$. Then the transitivity surface is given by the condition $u^{p}=$ const. The local coordinate system in the subspace $V_{r}$ will be denoted $\left[u^{\alpha}\right]$. Indexes $\alpha, \beta, \gamma, \sigma, \tau$ range from $n-r$ to $n-1$

There is a summation within the specified limits of index change on repeated upper and lower indexes. The subject of our study are the conditions for the existence of the algebra of first order symmetric operators (integrals of motion) of the classical and quantum motion equations for a charged scalar test particle in an external electromagnetic field.

\subsection{Hamilton-Jacobi Equation}

Let us consider the Hamilton-Jacobi equation for a charged test particle in an external electromagnetic field with the potential $A_{i}$ :

$$
g^{i j} P_{i} P_{j}=m, P_{i}=p_{i}+A_{i}, p_{i}=\frac{\partial \varphi}{\partial u^{i}} .
$$

It is commonly known that the first order integrals of motion of the free Hamilton-Jacobi equation are given by Killing vector fields $\xi_{\alpha}^{i}$ and have the following form

$$
Y_{\alpha}=\xi_{\alpha}^{i} p_{i}
$$

Let us show that in case if Equation (1) has $r$ independent first-order integrals of motion, these integrals have the form of (2). We will try to find the solution of the motion integrals in the form

$$
\hat{Y}_{\alpha}=\zeta_{\alpha}^{i} p_{i}
$$

Equation (1) allows the motion integrals of the form (3) if $H$ and $\hat{Y}_{\alpha}$ commute with respect to the Poisson brackets:

$$
\left[H, \hat{Y}_{\alpha}\right]_{P}=\frac{\partial H}{\partial p_{i}} \frac{\partial \hat{Y}_{\alpha}}{\partial x^{i}}-\frac{\partial H}{\partial x^{i}} \frac{\partial \hat{Y}_{\alpha}}{\partial p_{i}}=\left(g^{i l} \zeta_{\alpha, l}^{j}+g^{j l} \zeta_{\alpha, l}^{i}-g_{, l}^{i j} \zeta_{\alpha}^{l}\right) P_{i} P_{j}+2 g^{i \sigma}\left(\xi_{\alpha}^{j} F_{j i}+\left(\zeta_{\alpha}^{\beta} A_{\beta}\right)_{, i}\right) P_{\sigma}=0
$$

The functions $\zeta_{\alpha}^{j}$ satisfy the Killing equations:

$$
g^{i l} \tilde{\zeta}_{\alpha, l}^{j}+g^{j l} \xi_{\alpha, l}^{i}-g_{, l}^{i j} \xi_{\alpha}^{l}=0,
$$

and therefore they coincide with the Killing vector field:

$$
\zeta_{\alpha}^{j}=\xi_{\alpha}^{j}
$$


The coefficients before $P_{i} P_{j}$ in Equation (4) must vanish. Therefore, from Equation (4) it follows:

$$
\left(\xi_{\alpha}^{j} A_{j}\right)_{, i}=\xi_{\alpha}^{j} F_{i j}
$$

In contrast to the free Hamilton-Jacobi equation, the Equation (1) in the general case has no integrals of motion. The system of Equation (5) defines the set of admissible electromagnetic fields. In these fields, Equation (1) has $r$ first-order integrals of motion defined by the group $G_{r}$. The transitivity subspace where the group $G_{r}$ acts is given by the system of equations: We consider the following subsystem of the system (5):

$$
\left(\xi_{\alpha}^{j} A_{j}\right)_{, p}=\xi_{\alpha}^{j} F_{p j}
$$

Since in the chosen coordinate system $\xi_{\alpha, p}^{j}=0$, from Equation (5) it follows:

$$
\xi_{\alpha}^{\beta} A_{p, \beta}=0 \rightarrow A_{p}=A_{p}\left(u^{q}\right)
$$

Thus, the components $A_{p}$ can be made zero by the gradient transformation of the potential. Furthermore, we will select the calibration of the potential in exactly this way:

$$
A_{p}=0
$$

Then from (5) it follows:

$$
\left(\xi_{\alpha}^{\beta} A_{\beta}\right)_{, \gamma}=\xi_{\alpha}^{\beta} F_{\gamma \beta} .
$$

We prove that (8) is compatible. Indeed the system (8) can be present in the form

$$
\hat{Y}_{\alpha} \mathbf{A}_{\beta}=C_{\alpha \beta}^{\gamma} \mathbf{A}_{\gamma}, \mathbf{A}_{\beta}=\xi_{\beta}^{\alpha} A_{\alpha}
$$

Then the compatibility conditions can be transformed as follows:

$$
\left(\delta_{\alpha}^{\sigma}\left[\hat{Y}_{\beta} \hat{Y}_{\gamma}\right]-C_{\gamma \alpha}^{\sigma} \hat{Y}_{\beta}+C_{\beta \alpha}^{\sigma} \hat{Y}_{\gamma}\right) \mathbf{A}_{\sigma}=\left(C_{\alpha \beta}^{\tau} C_{\gamma \tau}^{\sigma}+C_{\beta \gamma}^{\tau} C_{\alpha \tau}^{\sigma}+C_{\gamma \alpha}^{\tau} C_{\beta \tau}^{\sigma}\right) \mathbf{A}_{\sigma}=0 .
$$

(The Bianchi identity is used.)

In [33] it was proved that the Hamilton-Jacobi and the Klein-Gordon-Fock equations admit a symmetry operator of the form

$$
\hat{Y}_{\alpha}=\xi_{\alpha}^{i}\left(\hat{p}_{i}+A_{i}\right)+\gamma_{\alpha}
$$

if and only if the electromagnetic field satisfies the system of equations

$$
\gamma_{\alpha, i}=\xi_{\alpha}^{j} F_{j i}
$$

By comparing Equations (5) and (6) one can show that:

$$
\gamma_{\alpha}=-\xi_{\alpha}^{\beta} A_{\beta}
$$

Therefore the operator (10) takes the form (2).

\subsection{Klein-Gordon-Fock Equation}

Let us consider the Klein-Gordon-Fock equation:

$$
\hat{H} \varphi=\left(g^{i j} \hat{P}_{i} \hat{P}_{j}\right) \varphi=m \varphi, \hat{P}_{j}=-\imath \hat{\nabla}_{i}+A_{i}
$$

$\hat{\nabla}_{i}$-operator of the covariant derivative, with metric-compatible connectivity, corresponding to the operator of the partial derivative $-\hat{\partial}_{i}=\imath \hat{p}_{i}$ with respect to the coordinate $u_{i} ; \varphi$ is a field of a scalar particle with mass $m$.

We denote the Laplase-Beltrami operator as $\hat{H}_{0}$ : 


$$
\hat{H}_{0}=g^{i j} \hat{\nabla}_{i} \hat{\nabla}_{j}
$$

Then the operator $\hat{H}$ can be presented as:

$$
\hat{H}=-\hat{H}_{0}+\hat{H}_{e} \cdot \hat{H}_{e}=2 A^{i} \hat{p}_{i}-\imath\left(\hat{\nabla}_{i} A^{i}\right)+A_{i} A^{i} .
$$

Let us prove the

Statement.

Algebra of symmetry operators of the Klein-Gordon-Fock equation coincides with the Lie algebra of the group $G_{r}$. That is the Klein-Gordon-Fock operator commutes with the operators

$$
\hat{Y}_{\alpha}=\xi_{\alpha}^{i} \hat{p}_{i}
$$

for the admissible external electromagnetic field.

Indeed the commutator of operators $\hat{H}$ and $\hat{p}_{i}$ has the form

$$
\left[\hat{H}, \hat{Y}_{\alpha}^{i}\right]=\left[\hat{H}_{0}, \hat{Y}_{\alpha}^{i}\right]+\left[\hat{H}_{e}, \hat{Y}_{\alpha}^{i}\right]=0 .
$$

As it is known,

$$
\left[\hat{H}_{0}, \hat{Y}_{\alpha}^{i}\right]=0 \rightarrow\left[g^{i j} \hat{\partial}_{i} \hat{\partial}_{j}+\left(g_{, j}^{i j}+g^{i j} \chi_{, j} \hat{\partial}_{i}\right), \xi_{\alpha}^{i} \hat{\partial}_{i}\right]=0 .
$$

We denoted here: $2 \chi_{i}=-g_{k l} g_{, i}^{k l}$. Since the functions $\xi_{\alpha}^{i}$ satisfy the Killing equations, from (16) we get the condition:

$$
g^{i l} \xi_{\alpha, i l}^{k}+\left(g_{, l}^{i l}+g^{i l} \chi_{, l}\right) \xi_{\alpha, i}^{k}-\left(g_{, l}^{i l}+g^{i l} \chi_{, l}\right),{ }_{i} \xi_{\alpha}^{i}=0 .
$$

Using the consequences from the Killing equations we get

$$
\begin{gathered}
g_{, i l}^{i l} \xi_{\alpha}^{k}=g^{i k} \xi_{\alpha, i l}^{l}+g_{, l}^{i l} \xi_{\alpha, i}^{k} \\
g^{i l} \chi_{l} \xi_{\alpha, i}^{k}-\left(g^{i l} \chi_{, l}\right)_{, i} \xi_{\alpha}^{i}=g^{i l}\left(\chi_{, l} \xi_{\alpha}^{i}\right)_{, i .} .
\end{gathered}
$$

It allows reducing (17) to the form

$$
\left(\xi_{\alpha, i}^{i}+\chi_{i} \xi_{\alpha}^{i}\right)_{, l}=0
$$

Since:

$$
2\left(\xi_{\alpha, i}^{i}+\chi_{i} \xi_{\alpha}^{i}\right)=\xi_{\alpha}^{i} g_{k l} g_{, i}^{k l}+2 \xi_{\alpha, i}^{i}=0,
$$

Equation (17) is reduced to an identity. Because the commutator $\left[\hat{H}_{e}, \hat{Y}_{\alpha}\right]$ equals to zero we obtain necessary and sufficient conditions for the existence of the symmetry operators algebra of Equation (13) in the form

$$
\begin{gathered}
A^{l} \xi_{\alpha, l}^{k}-A_{, l}^{k} \xi_{\alpha}^{l}=0 \rightarrow\left(\xi_{\alpha}^{\beta} A_{\beta}\right)_{, i}=\xi_{\alpha}^{\beta} F_{i \alpha} . \\
\xi_{\alpha}^{i}\left(A_{l} A^{l}\right)_{, i}=0 . \\
\xi_{\alpha}{ }^{k}\left(A_{, l}^{l}+A^{l} \chi_{, l}\right)_{, k}=0
\end{gathered}
$$

The Equation (19) is a consequence of Equation (18) and the Killing equations, because:

$$
\xi_{\alpha}{ }^{i} A_{l} A^{l}{ }_{, i}+\xi_{\alpha}{ }^{i} A^{l} A_{l, i}=A_{l} A^{k} \xi_{\alpha, k}^{l}+\xi_{\alpha}{ }^{i} A^{l} A_{l, i}=\left(\xi_{\alpha}^{\beta} A_{\beta}\right)_{, i}+\xi_{\alpha}^{\beta} F_{\beta i}=0 .
$$

Let us consider Equation (20). Using the condition (18) the first term can be presented as:

$$
\xi_{\alpha}^{k} A_{, k l}^{l}=\left(\xi_{\alpha}^{k} A^{l}, k\right)_{, l}-\xi_{\alpha, l}^{k} A^{l}{ }_{, k}=\left(A^{k} \xi_{\alpha, k l}^{l}+\xi_{\alpha, l}^{k} A_{, k}^{l}\right)-\xi_{\alpha, l}^{k} A_{, k}^{l}=A^{k} \xi_{\alpha, k l}^{l}
$$

The second term we transform as follows: 


$$
\left(A^{l} \chi_{l}\right)_{k} \xi_{\alpha}^{k}=A^{k}\left(\xi_{\alpha, k}^{l} \chi_{l}+\xi_{\alpha}^{l} \chi_{, l k}\right) \rightarrow A^{k}\left(\xi_{\alpha, l}^{l}+\xi_{\alpha}^{l} \chi_{, l}\right)_{, k}=0 .
$$

Thus only one condition (18) remains. The algebra exists, if and only if the admissible electromagnetic field exists.

The Statement is proved.

The integrals of motion of the Hamilton-Jacobi and the Klein-Gordon-Fock equations exist for the same electromagnetic fields and have the same form. To find the admissible electromagnetic fields, it is necessary to investigate the compatibility conditions of the system (6) with the form

$$
\gamma_{\sigma, \alpha i}-\gamma_{\sigma, i \alpha}=\xi_{\sigma}^{\beta} F_{i \alpha, \beta}+\xi_{\sigma, i}^{\beta} F_{\beta \alpha}-\xi_{\sigma, \alpha}^{\beta} F_{\beta i}=0 .
$$

The system (21) must be supplemented with the Bianchi identities. One can use the solutions $F_{i \alpha}$ to find the potential of the admissible electromagnetic field by integrating the compatible system of equations:

$$
A_{\alpha, \beta}-A_{\beta, \alpha}=F_{\beta \alpha} .
$$

We follow A. Z. Petrov [39]. This book contains all metrics of space-time manifolds in which the groups $G_{3}(N)$ act. We will hold to the notation accepted in this book with one exception - the non-ignored variables will be denoted by $u^{0}$. In addition for convenience we will use the notation $u^{i}=u_{i}$. Functions that depend only one the variables $u^{0}=u_{0}$ are denoted by by lowercase Greek letters with a single right subscript. Examples: $\alpha_{\alpha}=$ $\alpha_{\alpha}\left(u^{0}\right) ; \xi_{\beta}^{\alpha}=\xi_{\beta}^{\alpha}\left(u^{i}\right)$. Constants are denoted by by lowercase Latin letters with the tilda symbol. Derivatives with respect to the variables $u_{0}$ are denoted by dots. Example: $\dot{\alpha_{0}}=\partial \alpha / \partial u_{0}$.

\section{Solvable Groups $G_{3}(N)$}

According to the Bianchi classification, depending on the set of structural constants $C_{\alpha \beta}^{\gamma}$, there are nine types of groups $G_{3}(N)$. The first seven groups are solvable. Let us list them.

Solvable groups:

$$
\left\{\begin{array}{l}
G_{3}(I): C_{\alpha \beta}^{\alpha}=0 ; \\
G_{3}(I I): C_{12}^{\alpha}=0, C_{13}^{\alpha}=0 C_{23}^{\alpha}=\delta_{1}^{\alpha} ; \\
G_{3}(I I): C_{12}^{\alpha}=0, C_{13}^{\alpha}=\delta_{1}^{\alpha} C_{23}^{\alpha}=0 ; \\
G_{3}(I V): C_{12}^{\alpha}=0, C_{13}^{\alpha}=\delta_{1}^{\alpha} C_{23}^{\alpha}=\delta_{1}^{\alpha}+\delta_{2}^{\alpha} ; \\
G_{3}(V): C_{12}^{\alpha}=0, C_{13}^{\alpha}=\delta_{1}^{\alpha} C_{23}^{\alpha}=\delta_{2}^{\alpha} ; \\
G_{3}(V I): C_{12}^{\alpha}=0, C_{13}^{\alpha}=\delta_{1}^{\alpha} C_{23}^{\alpha}=q \delta_{2}^{\alpha} \cdot(q \neq 0,1) \\
G_{3}(V I I): C_{12}^{\alpha}=0, C_{13}^{\alpha}=\delta_{1}^{\alpha} C_{23}^{\alpha}=2 \delta_{2}^{\alpha} \cos \alpha, \alpha=\text { const. }
\end{array}\right.
$$

The groups $G_{3}(I)-G_{3}(V I I)$ contain the Abelian subgroup with the Killings vectors $\xi_{1}^{i}=\delta_{1}^{i}, \xi_{2}^{i}=\delta_{2}^{i}$. Therefore from (21) and from the Bianchi identities, it follows:

$$
F_{i j, 1}=F_{i j, 2}=F_{12,3}=F_{12,0}=0, F_{13,0}+F_{01,3}=0, F_{23,0}+F_{02,3}=0 .
$$

For the groups $G(I)-G(V I)$ the functions $\xi_{3}^{\alpha}, \xi_{3, i}^{\alpha}$ can be presented as the general formula:

$$
\xi_{3}^{\alpha}=\left(k u_{1}+\varepsilon u_{2}\right) \delta_{1}^{\alpha}+n u_{2} \delta_{2}^{\alpha}-\delta_{3}^{\alpha}, \xi_{3, i}^{\alpha}=\left(k \delta_{i 1}+\varepsilon \delta_{i 2}\right) \delta_{2}^{\alpha}+n \delta_{i 2} \delta_{2}^{i} .
$$

Parameters: $k, \varepsilon, n$ for each number $N$ take values:

$$
\begin{aligned}
& N=I \rightarrow k=n=\varepsilon=0, \\
& N=I I \rightarrow k=n=0, \varepsilon=1, \\
& N=I I \rightarrow k=1, n=\varepsilon=0, \\
& N=I V \rightarrow k=n=\varepsilon=1, \\
& N=V \rightarrow k=n=1, \varepsilon=0,
\end{aligned}
$$


$N=V I \rightarrow k=1, n=2, \varepsilon=0$.

The equation system (21) together with the Bianchi identities has the form

$$
\left\{\begin{array}{l}
F_{01,3}=k F_{01}, F_{02,3}=\varepsilon F_{01}+n F_{02}, F_{03,3}=0 \\
F_{13,3}=k F_{13}, F_{12,3}=(k+n) F_{12}, F_{23,3}=\varepsilon F_{13}+n F_{23} \\
F_{12,0}=F_{12,3}=0, F_{01,3}+F_{13,0}=0, F_{02,3}+F_{23,0}=0 .
\end{array}\right.
$$

For the group $G(V I I)$ the functions $\xi_{3}^{\alpha}, \xi_{3, i}^{\alpha}$ can be presented as follows:

$$
\xi_{3}^{\alpha}=-u_{2} \delta_{1}^{\alpha}+\left(2 u_{2} \cos \alpha+u_{1}\right) \delta_{2}^{\alpha}+\delta_{3}^{\alpha} \xi_{3, i}^{\alpha}=-\delta_{i 2} \delta_{1}^{\alpha}+\left(2 \delta_{i 2} \cos \alpha+\delta_{i 1}\right) \delta_{2}^{\alpha} .
$$

Formula (26) differ from ones listed in the book [39] in regard to the exchange:

$$
q=2 \cos \alpha,(\alpha=\text { const }) .
$$

Let us consider Equation (25) for each of the groups $G_{3}(I)-G_{3}(V I)$.

\subsection{The Group $G_{3}(I)$}

In this case $\xi_{3}^{i}=\delta_{3}^{i}$. From (25) it follows:

$$
F_{i j, 3}=0, F_{\alpha \beta, 0}=0 \rightarrow F_{\alpha 0}=\dot{\beta_{\alpha}}\left(u_{0}\right) .
$$

Potential $A_{i}$ is derived from the system of differential equation:

$$
A_{j, i}-A_{j, i}=F_{i j}
$$

and has the form

$$
A_{\alpha}=\beta_{\alpha}+\tilde{c}_{\alpha \beta} u^{\beta}, \tilde{c}_{\alpha \beta}=-\tilde{c}_{\beta \alpha}
$$

Using Equation (9), one can show that $\tilde{c}_{\alpha \beta}=0$.

\subsection{Group $G_{3}(I I)$}

In this case $k=n=0, \varepsilon=1$ and the system of Equation (25) have the form

$$
\left\{\begin{array}{l}
F_{12,3}=F_{12,0}=F_{13,3}=F_{03,3}=0 ; \\
F_{02,3}=F_{01}, F_{23,0}+F_{02,3}=0, F_{23,3}+F_{13}=0 ; \\
F_{13,0}+F_{01,3}=0, F_{23,0}+F_{02,3}=0 .
\end{array}\right.
$$

From the first equations if the system (29) it follows:

$$
F_{12}=\tilde{a}, F_{03}=\dot{\gamma}_{0}, F_{13}=2 \tilde{b} .
$$

By placing these equations in the remaining equations of the system (30) we get

$$
F_{01}=\dot{\alpha}_{0}, F_{02}=\dot{\alpha}_{0} u_{3}+\dot{\beta}_{0}, F_{23}=-\alpha_{0}+2 \tilde{b} u_{3} .
$$

A particular solution of the system (28) in the selected gauge has the form

$$
A_{1}=\alpha_{0}-2 \tilde{b} u_{3}, A_{2}=\alpha_{0} u_{3}+\beta_{0}+\tilde{a} u_{1}-\tilde{b} u_{3}^{2}, A_{3}=\gamma_{0} .
$$

Using Equation (9), one can show that $\tilde{a}=\tilde{b}=0$.

3.3. Group $G_{3}(I I I)$

In this case $k=0, n=\varepsilon=0$ and the systems of Equation (25) have the form

$$
\left\{\begin{array}{l}
F_{03,3}=F_{02,3}=F_{23,3}=F_{12,3}=F_{12,0}=0 \\
F_{01,3}=F_{01}, F_{12,3}=F_{12}, F_{13,3}=F_{13} \\
F_{23,0}+F_{02,3}=0, F_{13,0}+F_{01,3}=0, .
\end{array}\right.
$$


Hence we find the functions $F_{i j}$ :

$$
\begin{aligned}
& F_{01}=\dot{\alpha_{0}} \exp u_{3}, F_{02}=\dot{\beta_{0}}, F_{03}=\dot{\gamma_{0}}, \\
& F_{12}=0, F_{13}=-\alpha_{0} \exp u_{3}, F_{23}=\tilde{a} .
\end{aligned}
$$

A particular solution of the system (28) in the selected gauge has the form

$$
A_{1}=\alpha_{0} \exp u_{3}, A_{2}=\beta_{0}, A_{3}=\gamma_{0}+\tilde{a} u_{2} .
$$

Using Equation (9), one can show that $\tilde{a}=0$.

3.4. Group $G_{3}(I V)$

In this case $k=n=\varepsilon=1$ and the systems of Equation (25) have the form

$$
\left\{\begin{array}{l}
F_{01,3}=F_{01}, F_{02,3}=F_{01}+F_{02}, F_{03,3}=0, \\
F_{12,3}=2 F_{12}, F_{13,3}=F_{13}, F_{23,3}=F_{23}+F_{13}=0, \\
F_{13,0}+F_{01,3}=0, F_{23,0}+F_{02,3}=0, F_{01,3}+F_{13,0}=0, F_{12,3}=0 .
\end{array}\right.
$$

From here it follows that

$$
\begin{gathered}
F_{01}=\dot{\alpha_{0}} \exp u_{3}, F_{02}=\left(\dot{\alpha_{0}} u_{3}+\dot{\beta_{0}}\right) \exp u_{3}, F_{03}=\dot{\gamma}_{0}, \\
F_{12}=0, F_{13}=-\alpha_{0} \exp u_{3}, F_{23}=-\left(\alpha_{0} u^{3}+\alpha_{0}+\beta_{0}\right) \exp u_{3} .
\end{gathered}
$$

A particular solution of the system (28) in the selected gauge has the form

$$
A_{1}=\alpha_{0} \exp u_{3}, A_{2}=\left(\alpha_{0} u_{3}+\beta_{0}\right) \exp u_{3}, A_{3}=\gamma_{0} .
$$

3.5. Group $G_{3}(V)$.

In this case $k=n=1, \varepsilon=0$, and the systems of Equation (25) have the form

$$
\left\{\begin{array}{l}
F_{01,3}=F_{01}, F_{02,3}=F_{02}, F_{03,3}=0 ; \\
F_{12,3}=F_{12}, F_{13,3}=F_{13}, F_{23,3}=F_{23} \\
F_{13,0}+F_{01,3}=0, F_{23,0}+F_{02,3}=0, F_{12,3}=F_{12,0}=0 .
\end{array}\right.
$$

From here it follows:

$$
\begin{gathered}
F_{01}=\dot{\alpha_{0}} \exp u_{3}, F_{02}=\dot{\beta_{0}} \exp u_{3}, F_{03}=\dot{\gamma_{0}}, \\
F_{12}=0, F_{13}=-\alpha_{0} \exp u_{3}, F_{23}=-\beta_{0} \exp u_{3} .
\end{gathered}
$$

A particular solution of the system (28) in the selected gauge has the form

$$
A_{1}=\alpha_{0} \exp u_{3}, A_{2}=\beta_{0} \exp u_{3}, A_{3}=\gamma_{0} .
$$

3.6. Group $G_{3}(V I)$

In this case $k=1, n=2, \varepsilon=0$ and the systems of Equation (25) have the form

$$
\left\{\begin{array}{l}
F_{01,3}=F_{01}, F_{02,3}=2 F_{02}, F_{03,3}=0 ; \\
F_{12,3}=3 F_{12}, F_{13,3}=F_{13}, F_{23,3}=2 F_{23} \\
F_{12,0}=F_{12,3}=0, F_{13,0}+F_{01,3}=0, F_{23,0}+F_{02,3}=0, .
\end{array}\right.
$$

From here it follows:

$$
\begin{gathered}
F_{01}=\dot{\alpha_{0}} \exp u_{3}, F_{02}=\dot{\beta_{0}} \exp 2 u_{3}, F_{03}=\dot{\gamma_{0}}, \\
F_{12}=0, F_{13}=-\alpha_{0} \exp u_{3}, F_{23}=-2 \beta_{0} \exp 2 u_{3} .
\end{gathered}
$$


A particular solution of the system (26) in the selected gauge has the form

$$
A_{1}=\alpha_{0} \exp u_{3}, A_{2}=\beta_{0} \exp 2 u_{3}, A_{3}=\gamma_{0} .
$$

\subsection{Group $G_{3}(V I I)$}

In this case the relations (25) occur, and the systems of Equation (21) can be presented as:

$$
F_{i \alpha, 3}+\delta_{1 i} F_{2 \alpha}-\delta_{1 \alpha} F_{2 i}+\delta_{2 i}\left(2 F_{2 \alpha} \cos \alpha-F_{1 \alpha}\right)-\delta_{2 \alpha}\left(2 F_{2 i} \cos \alpha-F_{1 i}\right)=0 .
$$

Hence, using the Bianchi identities as well, we obtain the following system of equations:

$$
\left\{\begin{array}{l}
F_{01,3}+F_{02}=0, F_{02,3}+2 \cos \alpha F_{02}-F_{01}=0, F_{03,3}=0 \\
F_{12}=0, F_{13,3}+F_{23}=0, F_{23,3}+2 \cos \alpha F_{23}-F_{13}=0 \\
F_{13,0}+F_{01,3}=0, F_{23,0}+F_{02,3}=0
\end{array}\right.
$$

First of all, let us find the functions $F_{13}, F_{02}$ :

$$
\begin{aligned}
& F_{13}=\left(v_{0} \sin \left(u_{3} \sin \alpha\right)+\mu_{0} \cos \left(u_{3} \cos \alpha\right)\right) \exp \left(-u_{3} \cos \alpha\right), \\
& F_{02}=\left(\dot{\alpha_{0}} \sin \left(u_{3} \sin \alpha\right)+\dot{\beta_{0}} \cos \left(u_{3} \cos \alpha\right)\right) \exp \left(-u_{3} \cos \alpha\right),
\end{aligned}
$$

By placing them into the system's Equation (40), we find the relation between the functions $\alpha_{0}, \beta_{0}, v_{0}, \mu_{0}$ :

$$
\nu_{0}=\alpha_{0}, \mu_{0}=\beta_{0}
$$

following which, from the relations:

$$
F_{02}=-F_{01,3}, F_{23}=-F_{13,3}, F_{03}=0,
$$

we define the functions $F_{01}, F_{23}, F_{03}$ :

$$
\begin{gathered}
F_{23}=\left(\alpha_{0} \sin \left(-\alpha+u_{3} \sin \alpha\right)+\beta_{0} \cos \left(-\alpha+u_{3} \cos \alpha\right)\right) \exp \left(-u_{3} \cos \alpha\right), \\
F_{01}=\left(\dot{\alpha_{0}} \sin \left(\alpha+u_{3} \sin \alpha\right)+\dot{\beta_{0}} \cos \left(\alpha+u_{3} \cos \alpha\right)\right) \exp \left(-u_{3} \cos \alpha\right), F_{03}=\dot{\gamma_{0}}
\end{gathered}
$$

A particular solution of the system (28) in the selected gauge has the form

$$
\begin{gathered}
A_{1}=\left(\alpha_{0} \sin \left(\alpha+u_{3} \sin \alpha\right)+\beta_{0} \cos \left(\alpha+u_{3} \cos \alpha\right)\right) \exp \left(-u_{3} \cos \alpha\right), \\
A_{2}=\left(\alpha_{0} \sin \left(u_{3} \sin \alpha\right)+\beta_{0} \cos \left(u_{3} \cos \alpha\right)\right) \exp \left(-u_{3} \cos \alpha\right), A_{3}=\gamma_{0} .
\end{gathered}
$$

\section{Insolvable Groups $G_{3}(N)$}

Unsolvable groups $G_{3}(V I I I)$ and $G_{3}(I X)$ do not contain the Abelian subgroups and have more complex algebraic structures:

$$
\begin{gathered}
G_{3}(V I I I): C_{12}^{\alpha}=\delta_{1}^{\alpha}, C_{13}^{\alpha}=2 \delta_{2}^{\alpha} C_{23}^{\alpha}=-\delta_{3}^{\alpha} . \\
G_{3}(I X): C_{12}^{\alpha}=\delta_{3}^{\alpha}, C_{13}^{\alpha}=-\delta_{2}^{\alpha} C_{23}^{\alpha}=\delta_{1}^{\alpha} .
\end{gathered}
$$

For both structures the Killing vector field $\xi_{1}^{i}$ has the form

$$
\xi_{1}^{i}=\delta_{2}^{i}
$$

Therefore from the system (21) when $\sigma=1$ it follows:

$$
F_{i j, 2}=0 \text {. }
$$

Taking this condition into account, we consider the remaining equations of the system. 


\subsection{Group $G_{3}(V I I I)$}

Functions $\xi_{2}^{\alpha}, \xi_{3}^{\alpha}$ and their derivatives have the form

$$
\left\{\begin{array}{l}
\xi_{2}^{\alpha}=u_{2} \delta_{2}^{\alpha}+\delta_{3}^{\alpha}, \xi_{2, i}^{\alpha}=\delta_{i 2} \delta_{2}^{\alpha} \\
\xi_{3}^{\alpha}=\delta_{1}^{\alpha} \exp u_{3}+\delta_{2}^{\alpha} u_{2}^{2}+2 u_{2} \delta_{3}^{\alpha}, \xi_{3, i}^{\alpha}=\delta_{3 i} \delta_{1}^{\alpha} \exp u_{3}+2\left(u_{2} \delta_{2}^{\alpha}+\delta_{3}^{\alpha}\right) \delta_{2 i}
\end{array}\right.
$$

Because $F_{i j, 2}=0$, the system of Equation (21) splits into two

$$
\left\{\begin{array}{l}
F_{i \alpha, 3}+\left(\delta_{3 i} F_{1 \alpha}-\delta_{3 \alpha} F_{1 i}\right)+2\left(\delta_{2 i} 2 F_{3 \alpha}-\delta 2 \alpha F_{3 i}\right) \exp -u_{3}=0 \\
F_{i \alpha, 3}+\delta_{1 i} F_{2 \alpha}-\delta_{1 \alpha} F_{2 i}+\delta_{2 i}\left(2 F_{2 \alpha} \cos \alpha-F_{1 \alpha}\right)-\delta_{2 \alpha}\left(2 F_{2 i} \cos \alpha-F_{1 i}\right)=0 .
\end{array}\right.
$$

Hence, using the Bianchi identities as well, we obtain the following system of equations:

$$
\left\{\begin{array}{l}
F_{12,3}+F_{12}=0, F_{23,3}+F_{23}=0, F_{02,3}+F_{02}=0 \\
F_{02,1}+2 F_{03} \exp -u_{3}=0, F_{03,1}+F_{01}=0 \\
F_{12,1}+2 F_{13} \exp -u_{3}=0 F_{23,1}+F_{12}=0 \\
F_{01,3}=F_{01,1}=F_{13,3}=F_{13,1}=F_{03,3}=0 \\
F_{12,0}+F_{02,1}=0, F_{01,3}=F_{03,1}=0 \\
F_{02,3}+F_{23,0}=0, F_{12,3,0}+F_{23,1}=0 .
\end{array}\right.
$$

By integrating this system we get

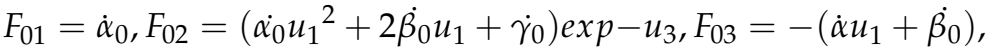

$$
\begin{aligned}
& F_{12}=2\left(\alpha_{0} u_{1}+\beta_{0}\right) \exp -u_{3}, F_{13}=-\alpha_{0}, F_{23}=\left(\alpha_{0} u_{1}^{2}+2 \beta_{0} u_{1}+\gamma_{0}\right) \exp -u_{3} \text {, }
\end{aligned}
$$

A solution of the system (26) in the selected gauge has the form

$$
A_{1}=\alpha_{0}, A_{2}=\left(\alpha_{0} u_{1}^{2}+2 \beta_{0} u_{1}+\gamma_{0}\right) \exp -u_{3}, A_{3}=-\left(\alpha_{0} u_{1}+\beta_{0}\right) .
$$

4.2. Group $G_{3}(I X)$

Functions $\xi_{2}^{\alpha}, \xi_{3}^{\alpha}$ and their derivatives have the form

$$
\left\{\begin{array}{l}
\xi_{2}^{\alpha}=\delta_{1}^{\alpha} \cos u_{2}+\left(\delta_{3}^{\alpha}-\delta_{2}^{\alpha} \cos u_{1}\right) \frac{\sin u_{2}}{\sin u_{1}}, \xi_{3}^{\alpha}=\frac{\partial \xi_{2}^{\alpha}}{\partial u_{2}} ; \\
\xi_{2, i}^{\alpha}=\sin u_{2}\left(-\delta_{i 2} \delta_{1}^{\alpha}+\frac{\delta_{i 1}}{\sin ^{2} u_{1}}\left(\delta_{2}^{\alpha}-\delta_{3}^{\alpha} \cos u_{1}\right)\right)+\left(\delta_{3}^{\alpha}-\delta_{2}^{\alpha} \cos u_{1}\right) \delta_{i 2} \frac{\cos u_{2}}{\sin u_{1}}
\end{array}\right.
$$

Because $F_{i j, 2}=0$, the system of Equation (21) splits into two subsystems:

$$
\left\{\begin{array}{l}
F_{i \alpha, 1} \sin u_{1}+\delta_{2 i}\left(F_{3 \alpha}-F_{2 \alpha} \cos u_{1}\right)-\delta_{2 \alpha}\left(F_{3 i}-F_{2 i} \cos u_{1}\right)=0 ; \\
F_{i \alpha, 3} \sin u_{1}+\left(\delta_{2 \alpha} F_{1 i}-\delta_{2 i} F_{1 \alpha}\right) \sin ^{2} u_{1}+\delta_{1 i}\left(F_{2 \alpha}-F_{3 \alpha} \cos u_{1}\right)-\delta_{1 \alpha}\left(F_{2 i}-F_{3 i} \cos u_{1}\right)=0 ;
\end{array}\right.
$$

Hence, using the Bianchi identities as well, we obtain the following systems of equations:

$$
\begin{aligned}
& \left\{\begin{array}{l}
F_{01,1}=F_{03,1}=F_{13,1}=F_{03,3}=0 \\
F_{01,3} \sin u_{1}+F_{02}-\cos u_{1} F_{03}=0 ; \\
F_{02,3}-F_{01} \sin u_{1}=0 ; \\
F_{12,3} \sin u_{1}+F_{23} \cos u_{1}=0 ; \\
F_{13,3} \sin u_{1}+F_{23}=0 ; \\
F_{23,3}-F_{13} \sin u_{1}=0 .
\end{array}\right. \\
& \left\{\begin{array}{l}
F_{02,1} \sin u_{1}-\cos u_{1} F_{02}+F_{03}=0 ; \\
F_{12,1} \sin u_{1}-\cos u_{1} F_{12}+F_{13}=0 ; \\
F_{23,1} \sin u_{1}-F_{23} \cos u_{1}=0 ;
\end{array}\right. \\
& \left\{\begin{array}{l}
F_{12,3}+F_{23,1}=0 ; F_{12,0}=F_{02,1} ; \\
F_{01,3}+F_{13,0}=0 ; F_{02,3}+F_{23,0}=0 .
\end{array}\right.
\end{aligned}
$$


From the system (47) we get

$$
\begin{gathered}
F_{13}=a_{1} \sin u_{3}+b_{1} \cos u_{3}, F_{23}=-\sin u_{1}\left(a_{1} \cos u_{3}-b_{1} \sin u_{3}\right), \\
F_{12}=\cos u_{1}\left(a_{1} \sin u_{3}+b_{1} \cos u_{3}\right)+c_{1},
\end{gathered}
$$

where $a_{1}, b_{1} c_{1}$ functions of variables $u_{0}, u_{1}$. Using the Bianchi identities (49) and rest equations of the system (47), (48), we get

$$
a_{1}=\alpha_{0} b_{1}=\beta_{0}, c_{1}=0
$$

Final solution can be present in the form

$$
\left\{\begin{array}{l}
F_{01}=\dot{\gamma_{0}}+\left(\dot{\alpha_{0}} \cos u_{3}-\dot{\beta_{0}} \sin u_{3}\right), \\
F_{02}=\left(\dot{\alpha_{0}} \sin u_{3}+\dot{\beta_{0}} \cos u_{3}\right) \sin u_{1}, \\
F_{03}=0 \\
F_{12}=\left(\alpha_{0} \sin u_{3}+\beta_{0} \cos u_{3}\right) \cos u_{1}, \\
F_{13}=\alpha_{0} \sin u_{3}+\beta_{0} \cos u_{3} \\
F_{23}=\left(-\alpha_{0} \cos u_{3}+\beta_{0} \sin u_{3}\right) \sin u_{1} .
\end{array}\right.
$$

A particular solution of the system (28) in the selected gauge has the form

$$
A_{3}=0, A_{1}=\gamma_{0}+\left(\alpha_{0} \cos u_{3}-\beta_{0} \sin u_{3}\right), A_{2}=\left(\alpha_{0} \sin u_{3}+\beta_{0} \cos u_{3}\right) \sin u_{1} .
$$

\section{Conclusions}

In conclusion, we note some ways for using the obtained results.

1. The considered metrics define homogeneous spaces, due to which the results are of interest in cosmology, especially when studying the processes occurring in the early stages of the Universe evolution.

2. The found external admissible fields, due to the special symmetry of homogeneous spaces, make it possible to construct interaction models of the axion field with the electromagnetic field, which is of interest when studying the problem of dark matter.

3. The results can be used to obtain exact self-consistent solutions in the General Theory of Relativity, in the scalar-tensor theory of gravity, in the Vaidya problem, as well as in the integration of field equations in other gravitational theories.

4. The results can be used to construct a theory of non-commutative integration of quantum motion equations in a strong gravitational field in the presence of fields of a gauge nature.

For the sake of convenience, we present all the obtained results in the Appendix A. For each group, the metric, electromagnetic potential, and integrals of motion are given. The metrics were found in [39]. We follow the notation used in this book. All functions $a_{\alpha \beta}$ depend only on the variable $u^{0}$ :

$$
a_{\alpha \beta}=a_{\alpha \beta}\left(u^{0}\right)
$$

Funding: This research received by Ministry of Sceince and High Education of Russian Federation, project FEWF-2020-0003.

Conflicts of Interest: The author declares no conflict of interest.

\section{Appendix A}

Appendix A.1. Group $\mathrm{G}_{3}(I)$

1. Metrics:

$$
d s^{2}=a_{\alpha \beta} d u^{\alpha} d u^{\beta}+e d u^{0^{2}}, e^{2}=1 .
$$

2. Potential of the admissible electromagnetic field:

$$
A_{0}=0, A_{\alpha}=\alpha_{\alpha} \alpha_{\alpha}=\alpha_{\alpha}\left(u^{0}\right) .
$$


3. Integrals of motion:

$$
\hat{Y}_{\alpha}=\hat{p}_{\alpha}
$$

Appendix A.2. Group $\mathrm{G}_{3}(I I)$

1. Metrics:

$$
\begin{gathered}
d s^{2}=d u^{1^{2}} a_{11}+2 d u^{1} d u^{2}\left(a_{12}+a_{11} u^{3}\right)+2 d u^{1} d u^{3} a_{13}+ \\
d u^{2^{2}}\left(a_{22}+2 a_{12} u^{3}+a_{11} u^{3^{2}}\right)+2 d u^{2} d u^{3}\left(a_{23}+a_{13} u^{3}\right)+ \\
+d u^{3^{2}} a_{33}+e d u^{3^{2}}, e^{2}=1 .
\end{gathered}
$$

2. Potential:

$$
A_{0}=0, A_{1}=\alpha_{0}, A_{2}=\alpha_{0} u_{3}+\beta_{0}, A_{3}=\gamma_{0} . .
$$

3. Integrals of motion:

$$
\hat{Y}_{1}=\hat{p}_{1}, \hat{\Upsilon}_{2}=\hat{p}_{2}, \hat{\Upsilon}_{3}=u^{2} \hat{p}_{1}-\hat{p}_{3} .
$$

Appendix A.3. Group $\mathrm{G}_{3}(I I I)$

1. Metrics:

$$
\begin{gathered}
d s^{2}=d u^{1^{2}} a_{11} \exp 2 u^{3}+2 d u^{1} d u^{2} a_{12} \exp u^{3}+2 d u^{1} d u^{3} a_{13} \exp u^{3}+ \\
2 d u^{2} d u^{3} a_{23}+d u^{2^{2}} a_{22}+d u^{3^{2}} a_{33}+e d u^{3^{2}}, e^{2}=1
\end{gathered}
$$

2. Potential of the admissible electromagnetic field:

$$
A_{0}=0, A_{1}=\alpha_{0} \exp u^{3}, A_{2}=\beta_{0}, A_{3}=\gamma_{0} .
$$

3. Integrals of motion:

$$
\hat{Y}_{1}=\hat{p}_{1}, \hat{Y}_{2}=\hat{p}_{2}, \hat{Y}_{3}=u^{1} \hat{p}_{1}-\hat{p}_{3}
$$

Appendix A.4. Group $\mathrm{G}_{3}(I V)$

1. Metrics:

$$
\begin{gathered}
d s^{2}=d u^{1^{2}} a_{11} \exp 2 u^{3}+2 d u^{1} d u^{2}\left(a_{12}+a_{11} u^{3}\right) \exp 2 u^{3}+2 d u^{1} d u^{3} a_{13} \exp u^{3}+ \\
2 d u^{2} d u^{3}\left(a_{23}+a_{13} u^{3}\right) \exp u^{3}+d u^{2^{2}}\left(a_{22}+2 a_{12} u^{3}+a_{11} u^{3^{2}}\right) \exp 2 u^{3} \\
+d u^{3^{2}} a_{33}+e d u^{3^{2}}, e^{2}=1 .
\end{gathered}
$$

2. Potential of the admissible electromagnetic field:

$$
A_{0}=0, A_{1}=\alpha_{0} \exp u^{3}, A_{2}=\left(\alpha_{0} u^{3}+\beta_{0}\right) \exp u^{3}, A_{3}=\gamma_{0} .
$$

3. Integrals of motion:

$$
\hat{Y}_{1}=\hat{p}_{1}, \hat{Y}_{2}=\hat{p}_{2}, \hat{Y}_{3}=\left(u^{2}+u^{1}\right) \hat{p}_{1}+u^{2} \hat{p}_{2}-\hat{p}_{3} .
$$

Appendix A.5. Group $\mathrm{G}_{3}(V)$

1. Metrics:

$$
\begin{gathered}
d s^{2}=d u^{1^{2}} a_{11} \exp 2 u^{3}+2 d u^{1} d u^{2} a_{12} \exp 2 u^{3}+2 d u^{1} d u^{3} a_{13} \exp u^{3}+ \\
2 d u^{2} d u^{3} a_{23} u^{3} \exp u^{3}+d u^{2^{2}} a_{22} \exp 2 u^{3}
\end{gathered}
$$




$$
+d u^{3^{2}} a_{33}+e d u^{3^{2}}, e^{2}=1 .
$$

2. Potential of the admissible electromagnetic field:

$$
A_{0}=0, A_{1}=\alpha_{0} \exp u^{3}, A_{2}=\beta_{0} \exp u^{3}, A_{3}=\gamma_{0} .
$$

3. Integrals of motion:

$$
\hat{Y}_{1}=\hat{p}_{1}, \hat{Y}_{2}=\hat{p}_{2}, \hat{Y}_{3}=u^{1} \hat{p}_{1}+u^{2} \hat{p}_{2}-\hat{p}_{3}
$$

Appendix A.6. Group $\mathrm{G}_{3}(V I)$

1. Metrics:

$$
\begin{gathered}
d s^{2}=d u^{1^{2}} a_{11} \exp 2 u^{3}+2 d u^{1} d u^{2} a_{12} \exp 3 u^{3}+2 d u^{1} d u^{3} a_{13} \exp u^{3}+ \\
2 d u^{2} d u^{3} a_{23} u^{3} \exp 2 u^{3}+d u^{2^{2}} a_{22} \exp 4 u^{3} \\
+d u^{3^{2}} a_{33}+e d u^{3^{2}}, e^{2}=1 .
\end{gathered}
$$

2. Potential of the admissible electromagnetic field:

$$
A_{0}=0, A_{1}=\alpha_{0} \exp u^{3}, A_{2}=\beta_{0} \exp 2 u^{3}, A_{3}=\gamma_{0} .
$$

3. Integrals of motion:

$$
\hat{\Upsilon}_{1}=\hat{p}_{1}, \hat{\Upsilon}_{2}=\hat{p}_{2}, \hat{\Upsilon}_{3}=u^{1} \hat{p}_{1}+2 u^{2} \hat{p}_{2}-\hat{p}_{3} .
$$

Appendix A.7. Group $\mathrm{G}_{3}(V I I)$

1. Metrics:

$$
\begin{gathered}
d s^{2}=d u^{1^{2}}\left[a_{11}+a_{12} \cos \left(2 u^{3} \sin \alpha\right)+a_{22} \sin \left(2 u^{3} \sin \alpha\right)\right] \exp \left(2 u^{3} \cos \alpha\right)+2 d u^{1} d u^{2}\left[a_{11} \cos \alpha+\left(a_{12} \cos \alpha+\right.\right. \\
\left.\left.+a_{22} \sin \alpha\right) \cos \left(2 u^{3} \sin \alpha\right)+\left(a_{22} \cos \alpha-a_{12} \sin \alpha\right) \sin \left(2 u^{3} \sin \alpha\right)\right] \exp \left(2 u^{3} \cos \alpha\right)+ \\
d u^{2^{2}}\left[a_{11}+\left(a_{12} \cos 2 \alpha+a_{22} \sin 2 \alpha\right) \cos \left(2 u^{3} \sin \alpha\right)+\left(a_{22} \cos 2 \alpha-a_{12} \sin 2 \alpha\right) \sin \left(2 u^{3} \sin \alpha\right)\right] \exp \left(2 u^{3} \cos \alpha\right)+ \\
2 d u^{1} d u^{3}\left[\left(a_{13} \cos \alpha-a_{23} \sin \alpha\right) \cos \left(u^{3} \sin \alpha\right)+\left(a_{13} \sin \alpha+a_{22} \cos \alpha\right) \sin \left(u^{3} \sin \alpha\right)\right] \exp \left(u^{3} \cos \alpha\right)+ \\
2 d u^{2} d u^{3}\left[a_{23} \sin \left(2 u^{3} \sin \alpha\right)+a_{13} \cos \left(2 u^{3} \sin \alpha\right)\right] \exp \left(u^{3} \cos \alpha\right)+d u^{3^{2}} a_{33}+e d u^{3^{2}}, e^{2}=1 .
\end{gathered}
$$

2. Potential of the admissible electromagnetic field:

$$
\begin{gathered}
A_{0}=0, A_{1}=\left(\alpha_{0} \sin \left(\alpha+u^{3} \sin \alpha\right)+\beta_{0} \cos \left(\alpha+u^{3} \cos \alpha\right)\right) \exp \left(-u^{3} \cos \alpha\right), \\
A_{2}=\left(\alpha_{0} \sin \left(u^{3} \sin \alpha\right)+\beta_{0} \cos \left(u^{3} \cos \alpha\right)\right) \exp \left(-u^{3} \cos \alpha\right), A_{3}=\gamma_{0} .
\end{gathered}
$$

3. Integrals of motion:

$$
\hat{Y}_{1}=\hat{p}_{1}, \hat{Y}_{2}=\hat{p}_{2}, \hat{Y}_{3}=-u^{2} \hat{p}_{1}+\left(2 u^{2} \cos \alpha+u^{1}\right) \hat{p}_{2}+\hat{p}_{3} .
$$

Appendix A.8. Group $\mathrm{G}_{3}($ VIII)

1. Metrics:

$$
\begin{gathered}
d s^{2}=d u^{1^{2}} a_{11}+2 d u^{1} d u^{2}\left(a_{11} u^{1^{2}}-2 a_{13} u^{1}+a_{12}\right) \exp -u^{3}+2 d u^{1} d u^{3}\left(a_{13}-a_{11} u^{1}\right)+ \\
d u^{2^{2}}\left[a_{22}-4 a_{23} u^{1}+2\left(a_{12}+2 a_{33}\right) u_{1}^{2}-4 a_{13} u^{1^{3}}+a_{11} u^{1^{4}}\right] \exp -2 u^{3} \\
+2 d u^{2} d u^{3}\left[a_{23}-\left(a_{12}+2 a_{33}\right) u_{1}+3 a_{13} u^{1^{2}}-a_{11} u^{1^{3}}\right] \exp -u^{3} \\
+2 d u^{3} 2\left(a_{11} u^{1^{2}}-2 a_{13} u^{1}+a_{33}\right)+e d u^{0^{2}}, e^{2}=1
\end{gathered}
$$


2. Potential of the admissible electromagnetic field:

$$
A_{0}=0, A_{1}=\alpha_{0}, A_{2}=\left(\alpha_{0} u_{1}^{2}+2 \beta_{0} u^{1}+\gamma_{0}\right) \exp -u^{3}, A_{3}=-\left(\alpha_{0} u^{1}+\beta_{0}\right) .
$$

3. Integrals of motion:

$$
\hat{Y}_{1}=\hat{p}_{2}, \hat{Y}_{2}=u^{2} \hat{p}_{2}+\hat{p}_{3}, \hat{Y}_{3}=\hat{p}_{1} \exp u^{3}+\hat{p}_{2} u^{2^{2}}+2 u^{2} \hat{p}_{3}
$$

Appendix A.9. Group $G_{3}(I X)$

\section{Metrics:}

$$
\begin{gathered}
d s^{2}=d u^{1^{2}}\left[a_{11}-\left(a_{12} \cos 2 u^{3}+a_{22} \sin 2 u^{3}\right)\right]+2 d u^{1} d u^{3}\left(\left(a_{13} \cos u^{3}-a_{23} \sin u^{3}\right)+\right. \\
+2 d u^{1} d u^{2}\left[\left(a_{13} \cos u^{3}-a_{23} \sin u^{3}\right) \cos u^{1}+\left(a_{12} \cos 2 u^{3}-a_{22} \sin 2 u^{3}\right) \sin u^{1}\right] \\
+d u^{2^{2}}\left[a_{33} \cos u^{1^{2}}+\left(a_{23} \cos u^{3}+a_{13} \sin u^{3}\right) \sin 2 u^{1}+\left(a_{12} \sin 2 u^{3}+a_{22} \cos 2 u^{3}+a_{11}\right) \sin u^{1^{2}}\right] \\
2 d u^{2} d u^{3}\left(a_{33} \cos u_{1}+\left(a_{23} \cos u^{3}+a_{13} \sin u^{3}\right) \sin u^{1}\right)+d u^{3^{2}} a_{33}+e d u^{02} .
\end{gathered}
$$

2. Potential of the admissible electromagnetic field:

$$
A_{0}=A_{3}=0, A_{1}=\left(\alpha_{0} \cos u^{3}-\beta_{0} \sin u^{3}\right), A_{2}=\left(\alpha_{0} \sin u^{3}+\beta_{0} \cos u^{3}\right) \sin u^{1} .
$$

3. Integrals of motion:

$$
\hat{Y}_{1}=\hat{p}_{2}, \hat{Y}_{2}=\hat{p}_{1} \cos u^{2}+\left(\hat{p}_{3}-\hat{p}_{2} \cos u^{1}\right) \frac{\sin u^{2}}{\sin u^{1}}, \hat{Y}_{3}=-\hat{p}_{1} \sin u^{2}+\left(\hat{p}_{3}-\hat{p}_{2} \cos u^{1}\right) \frac{\cos u^{2}}{\sin u^{1}}
$$

\section{References}

1. Stackel, P. Uber die intagration der Hamiltonschen differentialechung mittels separation der variablen. Math. Ann. 1897, 49, 145-147. [CrossRef]

2. Levi-Civita T. Sulla Integraziome Della Equazione Di Hamilton-Jacobi Per Separazione Di Variabili. Math. Ann. 1904, 59, 383-397. [CrossRef]

3. Jarov-Jrovoy, M.S. Integration of Hamilton-Jacobi equation by complete separation of variables method. J. Appl. Math. Mech. 1963, $27,173-219$.

4. Shapovalov, V.N. Symmetry of motion equations of free particle in riemannian space. Sov. Phys. J. 1975, 18, 1650-1654. [CrossRef]

5. Shapovalov, V.N. Symmetry and separation of variables in the Hamilton-Jacobi equation. Sov. Phys. J. 1978, 21, 1124-1132. [CrossRef]

6. Shapovalov, V.N. Stackel's spaces. Sib. Math. J. 1979, 20, 1117-1130. [CrossRef]

7. Bagrov, V.G.; Obukhov, V.V. Complete separation of variables in the free Hamilton-Jacobi equation. Theor. Math. Phys. 1993, 97, 1275-1289. [CrossRef]

8. Benenti, S. Separability in Riemannian Manifolds. SIGMA 2016, 12, 013. [CrossRef]

9. Miller, W. Symmetry And Separation of Variables; Cambridge University Press: Cambridge, UK, 1984; p. 318.

10. Obukhov, V.V. Hamilton-Jacobi equation for a charged test particle in the Stackel space of type (2.0). Symmetry 2020, $12,1289$. [CrossRef]

11. Obukhov, V.V. Integration of the Hamilton-Jacobi and Maxwell equations for Diagonal metrics. Russ. Phys. J. 2020, 63, 33-35. [CrossRef]

12. Obukhov, V.V. Hamilton-Jacobi equation for a charged test particle in the Stackel space of type (2.1). Int. J. Geom. Meth. Mod. Phys. 2020, 17, 2050186. [CrossRef]

13. Obukhov, V.V. Separation of variables in Hamilton-Jacobi and Klein-Gordon-Fock equations for a charged test particle in the stackel spaces of type (1.1). Int. J. Geom. Meth. Mod. Phys. 2021, 18, 2150036. [CrossRef]

14. Bagrov, V.G.; Obukhov, V.V. Separation of variables for the Klein-Gordon equation in special staeckel space-times. Class. Quant. Grav. 1990, 7, 19-25. [CrossRef]

15. Bagrov, V.G.; Obukhov, V.V. Shapovalov A.V. Special Stackel electrovac spacetimes. Pramana J. Phys. 1986, 26, 93-108. BF02847629. [CrossRef]

16. Bagrov, V.G.; Obukhov, V.V. Classes of exact solutions of the Einstein-Maxwell equations. Ann. Phys. 1983, B40, 181-188. [CrossRef]

17. Carter, B. New family of Einstein spaces. Phys. Lett. 1968, 26, 399-400. [CrossRef]

18. Osetrin, E.; Osetrin, K. Pure radiation in space-time models that admit integration of the eikonal equation by the separation of variables method. J. Math. Phys. 2017, 58, 112504. [CrossRef] 
19. Osetrin, K.E.; Filippov, A.E.; Osetrin, E.R. The spacetime models with dust matter that admit separation of variables in HamiltonJacobi equations of a test particle. Mod. Phys. Lett. A 2016, 31, 410. [CrossRef]

20. Osetrin, K.; Osetrin, E. Shapovalov wave-like spacetimes. Symmetry 2020, 12, 1372. [CrossRef]

21. Maharaj, S.D.; Goswami, R.; Chervon, S.V.; Nikolaev, A.V. Exact solutions for scalar field cosmology in f(R) gravity. Mod. Phys. Lett. A 2017, 32, 1750164. [CrossRef]

22. Rajaratnam, K.; Mclenaghan, R.G.; Valero, C. Orthogonal separation of the Hamilton Jacobi equation on spaces of constant curvature. SIGMA 2016, 12, 30. [CrossRef]

23. Rajaratnam, K.; Mclenaghan, R.G. Classification of Hamilton-Jacobi separation in orthogonal coordinates with diagonal curvature. J. Math. Phys. 2014, 55, 083521. [CrossRef]

24. McLenaghan, R.G.; Rastelli, G.; Valero, C. Complete separability of the Hamilton-Jacobi equation for the charged particle orbits in a Lienard-Wiehert field. J. Math. Phys. 2020, 61, 122903. [CrossRef]

25. Gray, F.; Houri, T.; Kubiznak, D.; Yasui, Y. Symmetry operators for the conformal wave equation. arXiv 2021, arXiv:2101.06700.

26. Bamba, K.; Capozziello, S.; Nojiri, S.; Odintsov, S.D. Dark energy cosmology: The equivalent description via different theoretical models and cosmography tests. Astrophys. Space Sci. 2012, 342, 155. [CrossRef]

27. Capozziello, S.; De Laurentis, M.; Odintsov, D. Hamiltonian dynamics and Noether symmetries in extended gravity cosmology. Eur. Phys. J. 2012, C72, 2068. [CrossRef]

28. Makarenko, A.N.; Obukhov, V.V. Exact solutions in modified gravity models. Entropy 2012, 14, 1140-1153. [CrossRef]

29. Akdemir, A.O.; Butt, S.I.; Nadeem, M.; Ragusa, M.A. New General Variants of Chebyshev Type Inequalities via Generalized Fractional Integral Operators. Mathematics 2021, 9, 122. [CrossRef]

30. Shapovalov, A.V.; Shirokov, I.V. Noncommutative integration method for linear partial differential equations. functional algebras and dimensional reduction. Theoret. Math. Phys. 1996, 106, 3-15. [CrossRef]

31. Magazev, A.A.; Shirokov, I.V.; Yurevich, Y.A. Integrable magnetic geodesic flows on Lie groups. Theor. Math. Phys. 2008, 156, 1127-1140. [CrossRef]

32. Magazev, A.A. Constructing a complete integral of the hamilton-jacobi equation on pseudo-riemannian spaces with simply transitive groups of motions. Math. Phys. Anal. Geom. 2021, 24, 11. [CrossRef]

33. Magazev A.A. Integrating Klein-Gordon-Fock equations in an extremal electromagnetic field on Lie groups. Theor. Math. Phys. 2012, 173, 1654-1667. [CrossRef]

34. Shapovalov, A.V.; Breev, A.I. Symmetry operators and separation of variables in the $(2+1)$-dimensional Dirac equation with external electromagnetic field. Int. J. Geom. Meth. Mod. Phys. 2018, 15, 1850085. [CrossRef]

35. Shapovalov, A.V.; Breev, A.I. The Dirac equation in an external electromagnetic field: Symmetry algebra and exact integration. J. Phys. Conf. Ser. 2016, 670, 012015.

36. Breev, A.I.; Shapovalov, A.V. Noncommutative Integrability of the Klein-Gordon and Dirac equation in (2+1)-dimentional spacetime. Russ. Phys. J. 2017, 59, 1956-1961. [CrossRef]

37. Shapovalov, A.; Breev, A. Non-commutative integration of the Dirac equation in homogeneous spaces. Symmetry 2020, $12,1867$. [CrossRef]

38. Makarenko, A.N.; Obukhov, V.V.; Osetrin, K.E. Integrability of Einstein-Weyl equations for spatially homogeneous models of type III by Bianchi. Russ. Phys. J. 2002, 45, 49-55. [CrossRef]

39. Petrov, A.Z. Einstein Spaces; Elsevier: Oxford, UK, 1969. 